T. Fülöp, C. Franceschi, K. Hirokawa, G. Pawelec (eds)

\section{Handbook on Immunosenescence: Basic Understanding and Clinical Applications}

Springer Science + Business Media, Dordrecht, 2009

ISBN 978-1-4020-9062-2

e-ISBN 978-1-4020-9063-9

The Handbook on Immunosenescence, a 1,650-page 2-volume encyclopedia on aging research with 78 articles, includes $5 \mathrm{sec}$ tions: (1) Methods and Models for Studying Immunosenescence; (2) Cellular Immunosenescence; (3) Mechanisms; (4) Clinical Relevance in Disease States, and (5) Modulation. Its strength is the broad coverage of aging, the immune system, and diseases of the elderly. For this reason, the Handbook of Immunosenescence is a worthy addition to any medical library.

The editors, however, get off to a rocky start in their preface entitled 'What Is Immunosenescence?' They write: 'We perceive a great need to assemble this current knowledge in one (sic) volume by collecting contributions from the most eminent researchers in the field from all around the world. In this way, we aim to facilitate a synthesis...' Unfortunately, the hoped-for 'synthesis' did not develop spontaneously and one wonders whether an editorial overview preceding each of the sections might have facilitated the desired synthesis by relating one article to others in the Handbook. In addition, the editors were sadly not well served by the publisher who produced two volumes with contributions that lacked consistency in the form or style of the text or references. Finally, the numerous typographical errors and inadequate index should be corrected in the next printing.

The first section on Methods and Models to study aging includes valuable articles on longitudinal studies of humans and short- and long-lived mouse strains. Two articles in this section are commended. One by Wikby et al. reports a longitudinal study of humans over 80 years of age from which an immune risk profile was defined based on T-cell number and function, evidence of inflammation, and persistent cytomegalovirus infection that predicted shorter longevity. The second by Yu et al. describes the characteristics of murine strains with shorter or longer lifespans.

The second section titled Cellular Immunosenescence, the longest and best integrated in the Handbook, is largely devoted to the aging of $\mathrm{T}$ cells. Lamar et al. discuss human age-associated decreased T-cell production following thymic involution that leads to a decreased diversity in the T-cell repertoire. This and the resultant holes in the $\mathrm{T}$-cell repertoire lead to impaired responses of $\mathrm{T}$ cells to immunogens and pathogens. Nikolich-Zugich and Long extend this theme in aging mice showing that age-associat- ed decreases in the diversity of the T-cell repertoire is associated with T-cell clonal expansions of the CD8 T-cell population. Some of the clonal T-cell expansions are specific for virus determinants expressed by cytomegalovirus. An interesting question is whether age-associated holes in the T-cell repertoire result from a 'crowding-out' by expanded T-cell specificities that share the same $\mathrm{T}$-cell receptor family that may be induced and maintained by persistent antigenic stimulation. Clambey et al. describe the IL-7- and 15-dependent clonal expansion of splenic CD8 T cells specific for lymphochoriomeningitis virus infection in mice. This clone can make up $80 \%$ of the CD8 splenic T cells. Despite the long persistence of CD8 clonal expansions there is no evidence that these cells in mice or in humans transform into neoplasms. Finally, Sikora and Brzezinska consider the possibility that impaired activation-induced T-cell death may contribute to the persistent lymphocyte T-cell clonal expansions that occur with aging. Unfortunately, there is no consensus that cell susceptibility to activation-induced death increases, decreases, or remains unchanged during aging. The next largest part of the second section is devoted to age-associated changes in the B-cell repertoire of mice by Scholz et al. and in humans by Gibson and Dunn-Walters. $\mathrm{B}$ cells, like T cells, suffer a loss of repertoire diversity with age. Not only may these losses of repertoire diversity appear to be associated with increased susceptibility to determinants on pathogens lost from the lymphocyte repertoire but also they may contribute to the origin of benign and malignant clonal B-cell expansions. The third major cellular element in the immune system, antigen-presenting cells, is considered by Agrawal et al., who conclude that these cells are relatively unchanged during aging. Similarly, the effect of aging on neutrophils, monocytes, natural killer cells and hematopoietic stem cells, although less studied, appears to have less consequence for the aging host - although when stressed these cell populations may also reveal impaired age-associated function. For example the competence of hematopoietic stem cells from old compared to young animals is revealed only when their capacity to regenerate progeny cells is stressed upon repeated transplantation. It is possible that age-associated defects are revealed in many cell types only under the stress of external or internal challenges. Whether cellular reserve is compromised by disease or stem cell exhaustion is not discussed. The second section ends with three articles on genetic polymorphisms of immune function that may be associated with lifespan.

The third section, entitled Mechanisms, reviews theories of aging. Age-associated immune decline has been related to the dysfunction in old lymphocytes of cytokine production, receptor expression or function, mitochondria, and proteosomes. However, the most convincing case is presented by Hakim in support of

\section{KARGER}

Fax +41613061234 E-Mail karger@karger.ch www.karger.com
() 2010 S. Karger AG, Basel

0304-324X/10/0563-0359\$26.00/0

Accessible online at:

www.karger.com/ger 
thymic involution as the pacesetter of immune senescence. This chapter includes a discussion of thymic involution and its consequences as well as current strategies that may prevent or reverse its occurrence. The other major theme is presented by Franceschi et al. who see immune senescence and the diseases of aging as caused by low-grade, chronic inflammation, termed 'InflammAging', and manifested by increased plasma levels of IL-1, IL-6 and TNF- $\alpha$. However, it remains unclear whether increases in inflammatory cytokines contribute to or result from the major diseases of aging.

The fourth section, devoted to the Clinical Relevance of immune senescence, might surprise geriatricians as its discussions of infectious disease focus on AIDS in two of the four articles that concern infection while common infections of the elderly, e.g. influenza, pneumonia, herpes zoster and urinary tract infection, are not considered although they are discussed in the fifth section by McElhaney (see below). Bryl and Witkowski correct the common confusion that autoimmune disease increases with age when in fact it is the frequency of autoantibodies that increases during aging. Another apparent paradox is presented by Henderson et al. and Robert et al. that immunizing animals with molecules from the atherosclerotic lesion can both induce atherosclerosis and prevent its development. In a critical review Sportes and Hakim offer compelling evidence that cancer incidence increases with age, as does immune senescence, but note that this association 'has lead (sic) to erroneous interpretation'. In fact, patients with primary immune deficiencies with impaired T-cell function have an increased incidence of lymphoid malignancies but not the most common tumors such as lung, prostate and colon, although the latter appear to be increased in immunosuppressed recipients of organ grafts. Gravekamp presents evidence that cancer vaccination prior to induction of cancer is more effective in young than old experimental animals but this situation is more complex when vaccination is attempted in young or old tumor-bearing animals. Kitagawa and Hirokawa offer a chapter that reviews experimental and clinical carcinogenesis with a useful discussion of the balance between cell proliferation and apoptosis as being two sides of the same coin - the former contributing to carcinogenesis, the latter to aging.
The fifth section discusses possible means to correct malnutrition, in general, and specific changes in zinc, selenium, and lipids that increase oxidative stress and decrease T-cell function. McElhaney and McGavin discuss the benefits of prophylactic vaccines against infectious diseases of the elderly including influenza, herpes zoster and pneumococcal vaccines as well as the potential for a therapeutic vaccine for Alzheimer's diseases. Mitchell and Aspinall discuss the role of IL-7 and other growth factors to sustain thymic function, and Fletcher et al. consider sex hormone 'ablation' to achieve the same goal. The section concludes with two chapters directed toward the future. Ritz and Gardner conclude that to date the results of nutraceutical administration for immune restoration are 'largely inconsistent'. Chen et al. offer the promise of gene therapy and cite the reported TERT gene-mediated reversal of shortened telomere length and lifespan of fibroblasts from patients with dyskeratosis congenita. It is fair to say that we will be hearing more on gene therapy research in the next edition of the Handbook.

The Handbook closes with an enigmatic one-page coda entitled 'Perspectives: Is Immunosenescence Clinically Relevant?' The editors write: 'Having read this book one can legitimately ask the question is immunosenescence clinically relevant and if it is so, what can be done for prevention, intervention and cure.' The editors appear to answer this question in the negative, '...what we do not know is that the changes in the immune response lead to various diseases...' Many experts in geriatrics and gerontology would take a more positive view of the 'clinical applications' resulting from the growing knowledge of immunosenescence. A good example is the recently licensed herpes zoster vaccine that reduces the risk of herpes zoster and post-herpetic neuralgia by more than $50 \%$ in elderly persons. Furthermore, the pharmaceutical industry has committed large sums of money to develop immune therapies of Alzheimer's disease and atherosclerosis showing their conviction that greater understanding of immunosenescence will lead to immunotherapies for the diseases of aging.

Dr. Marc E. Weksler, New York 